\title{
Optimal Fraction Repetition Codes for Access-Balancing in Distributed Storage
}

\author{
Wenjun Yu, Xiande Zhang and Gennian Ge
}

\begin{abstract}
To solve the access-balancing problem in distributed storage systems, we introduce a new combinatorial model, called MinVar model for fractional repetition (FR) codes. Since FR codes are based on graphs or set systems, our MinVar model is characterized by the property that the variance among the sums of block-labels incident to a fixed vertex is minimized. This characterization is different from Dau and Milenkovic's MaxMinSum model, while the minimum sum of labels is maximized. We show that our MinVar model is meaningful by distinguishing labelings with different variances but with the same MaxMin value for some FR codes. By reformulating the MinVar model to an equivalent vertex-labeling problem of graphs, we find several families of optimal FR codes with balanced access frequency, and provide fundamental results for both problems. It is interesting that MinVar model is closely related to the concept of magic-labeling in graph theory.
\end{abstract}

Keywords: Distributed storage; access-balance; fractional repetition codes; access-variance; magic-labeling

\section{INTRODUCTION}

Motivated by the access-balancing issue in the coding for distributed storage systems [11], Dau and Milenkovic [10] introduced a problem of labeling the points of the underlying combinatorial designs. In this framework, a file is split into several equal-sized parts and encoded into data chunks by an outer MDS code. After this, each data chunk is replicated a certain number (replication number) of times and distributed among multiple storage nodes based on an inner fractional repetition $(F R)$ code [20], [21], [24]. The combination of the outer MDS code and the inner FR code supports redundancy and reparability of the storage system, and constitutes a class of minimum bandwidth regenerating (MBR) codes [11] with the property of exact repair by transfer. The problem of balancing data placements and loads of the storage in such a scheme, requires a constant data replication number and a constant node volume of the inner FR code. This is the main reason that combinatorial designs such as Steiner systems are commonly employed in data placement [17], [18], [30]. Systems like Hadoop Distributed File System and Google File System apply this strategy [8].

Access balancing aims to balance the access requests to the nodes by using data chunk popularity information [6]. In Dau and Milenkovic's model [10], the data chunks are labeled by popularity, and the overall popularities of chunks stored on each node need to be balanced. That is, to find a proper labeling of the underling combinatorial design such that the sums of labels in each block are as equal as possible. In particular, they defined functions of designs to measure this property, MaxMin (or MinMax), the maximum (minimum) value of the minimum (maximum) block-sum in the design, and successfully found all Steiner triple systems that achieve the MaxMin value. This problem was further studied in [4] for Kirkman systems and in [5] for partial Steiner systems.

Although combinatorial designs are commonly used as underlying structures of the storage scheme, they are not usually the best choice for FR codes in general. Regarding to the maximum size of the file that can be stored in a DRESS code [19], [21], Silberstein and Etzion [26] studied optimal FR codes based on graphs and designs. They constructed two kinds of optimal FR codes with replication number two, one is based on Turán graphs and the other is based on graphs with large girth. For bigger replication number, they showed that transversal designs and generalized polygons can produce optimal FR codes.

In this paper, we focus on the access-balancing problem for optimal FR codes. By observing that the MaxMinSum model only cares about the minimum sum of labels, we introduce a new model which considers the variance of the sums of labels. The new model is called MinVar model, which aims to approach the minimum access-variance of overall popularities among all nodes. This is in fact a block-labeling problem of set systems, such that the variance of the sums of labels of blocks incident to any fixed vertex is minimized. When the minimum variance attains zero, the problem is indeed a magic labeling problem for graphs, from which we can find several optimal FR codes with balanced access requests. Our second contribution is introducing an equivalent problem, which is a vertex-labeling problem of graphs when the set system is linear. By solving this problem for special graphs, we estimate the minimum access-variance of the MinVar model for several optimal FR codes.

The paper is organized as follows. Section [reviews FR codes, set systems, graphs and their relations. Section [iI introduces the MinVar model, its relation to the magic labeling problem, and its equivalent vertex-labeling problem of graphs. In Section IV

W. Yu (yuwen jun@mail.ustc.edu.cn) and X. Zhang (drzhangx@ustc.edu.cn) are with School of Mathematical Sciences, University of Science and Technology of China, Hefei, 230026, Anhui, China. The research of X.Zhang was supported by the National Natural Science Foundation of China under Grant No. 11771419.

G. Ge (gnge@zju.edu.cn) is with the School of Mathematical Sciences, Capital Normal University, Beijing 100048, China. The research of G. Ge was supported by the National Natural Science Foundation of China under Grant No. 11971325, National Key Research and Development Program of China under Grant Nos. 2020YFA0712100 and 2018YFA0704703, and Beijing Scholars Program. 
we solve the equivalent vertex-labeling problem for several graphs, which helps to attack the MinVar problem in Section $\mathrm{V}$ Concluding remarks are provided in Section VI

\section{Preliminaries AND Notations}

In this section we provide useful definitions of codes, graphs and set systems, and their relations among each other.

\section{A. Fractional repetition codes}

El Rouayheb and Ramchandran [21] introduced the concept of DRESS (Distributed Replication based Exact Simple Storage) code, which consists of the concatenation of an outer MDS code and an inner FR code.

Let $[\theta]:=\{1,2, \ldots, \theta\}$. Assume that $n, \alpha, \theta, \rho$ are positive integers satisfying $n \alpha=\theta \rho$. An $(n, \alpha, \rho)$ FR code $\mathcal{C}$ is a collection of $n$ subsets of $[\theta], N_{1}, N_{2}, \ldots, N_{n}$, each of size $\alpha$ such that, each symbol of $[\theta]$ appears in exactly $\rho$ subsets of $\mathcal{C}$. A $[(\theta, M), k,(n, \alpha, \rho)]$ DRESS code is a code consisting of an outer $(\theta, M)$ MDS code and an inner $(n, \alpha, \rho)$ FR code $\mathcal{C}$. First, a file $\mathbf{f}=\left(x_{1}, x_{2}, \cdots, x_{M}\right) \in \mathbb{F}_{q}^{M}$ is encoded by the outer MDS code, and outputs a codeword $y_{\mathbf{f}}=\left(y_{1}, y_{2}, \cdots, y_{\theta}\right)$. Second, every symbol of $y_{\mathbf{f}}$ is placed on $n$ storage nodes using a way defined by $\mathcal{C}$ : place the symbol $y_{i}$ in the $j$ th node if $i \in N_{j}$ in $\mathcal{C}$. The definition of FR code ensures that each node stores exactly $\alpha$ symbols, and each symbol is placed on exactly $\rho$ nodes.

A valid DRESS code should have the following two properties. First, when some node $j$ fails, it is possible to find a set of $d=\alpha$ other nodes, such that each node passing exactly one symbol is able to repair node $j$. The repair bandwidth $d$ is the same as the repair bandwidth of an MBR code. Second, the stored file should be reconstructed from any set of $k$ nodes, which requires $\min _{|I|=k}\left|\cup_{i \in I} N_{i}\right| \geq M$ due to the property of the outer MDS code. Note that one can assume that $M=M(k)=\min _{|I|=k}\left|\cup_{i \in I} N_{i}\right|$ for a given DRESS code.

To maximize the file size and ensure correct reconstruction and repair, one can require that $\left|N_{i} \cap N_{j}\right| \leq 1$ for all $i \neq j$ [20]. Let $A(n, k, \alpha, \rho)$ be the maximum file size $M(k)$ among all $[(\theta, M), k,(n, \alpha, \rho)]$ DRESS codes, which indeed only depends on the inner FR code. Two upper bounds on $A(n, k, \alpha, \rho)$ were given in [21],

$$
\begin{gathered}
A(n, k, \alpha, \rho) \leq\left\lfloor\frac{n \alpha}{\rho}\left(1-\frac{\left(\begin{array}{c}
n-\rho \\
k
\end{array}\right)}{\left(\begin{array}{c}
n \\
k
\end{array}\right)}\right)\right\rfloor \text { and } \\
A(n, k, \alpha, \rho) \leq \varphi(k), \text { where } \varphi(1)=\alpha, \varphi(k+1)=\varphi(k)+\alpha-\left\lceil\frac{\rho \varphi(k)-k \alpha}{n-k}\right\rceil .
\end{gathered}
$$

An FR code is called $k$-optimal if $\min _{|I|=k}\left|\cup_{i \in I} N_{i}\right|=A(n, k, \alpha, \rho)$ for a given $k$, and is optimal if it is $k$-optimal for all $k \leq \alpha$.

Silberstein and Etzion [26] constructed two kinds of optimal FR codes with $\rho=2$, one is based on Turán graphs and the other is based on graphs with large girth. For $\rho>2$, they showed that transversal designs and generalized polygons produce optimal FR codes.

\section{B. Set Systems and Graphs}

For a finite set $V$ of points, let $\left(\begin{array}{l}V \\ r\end{array}\right)$ denote the set of all $r$-subsets of $V$. The pair $\mathcal{S}=(V, E)$ is called an $r$-uniform set system if $E \subset\left(\begin{array}{l}V \\ r\end{array}\right)$. The elements of $E$ are called blocks. The order of $\mathcal{S}$ is the number of points $|V|$, and the size of $\mathcal{S}$ is the number of blocks $|E|$. Such a pair $(V, E)$ is also known as a graph if $r=2$, where $V$ and $E$ are commonly referred to vertices and edges, respectively. When $r>2, \mathcal{S}$ is known as an $r$-uniform hypergraph (or $r$-graph), where elements of $E$ are referred to hyperedges. A set system is called linear if any two blocks intersect on at most one common point. The 2-shadow of $\mathcal{S}=(V, E)$ is a 2-uniform set system denoted by $\partial_{2} \mathcal{S}=\left(V^{\prime}, E^{\prime}\right)$, where $V^{\prime}=V, E^{\prime}=\{\{a, b\}:\{a, b\} \subseteq B, B \in E\}$. Clearly, if $\mathcal{S}$ is a 2-uniform set system then $\partial_{2} \mathcal{S}=\mathcal{S}$.

Two points $x, y \in V$ are adjacent in $\mathcal{S}$, if there exists a block $e \in E$, such that $\{x, y\} \subset e$. A point $x$ is incident with a block $e \in E$, if $x \in e$. The degree of $x$ is the number of blocks incident with $x$, denoted by $d(x)$. A set system is said to be $d$-regular if $d(x)=d$ for all $x \in V$, where $d$ is a positive integer. The incidence matrix $I(\mathcal{S})$ of a set system $\mathcal{S}=(V, E)$ is a binary $|V| \times|E|$ matrix with rows and columns indexed by $V$ and $E$, respectively, such that $I(\mathcal{S})_{i, e}=1$ if and only if $i \in e$. The line graph $L(\mathcal{S})$ of a set system $\mathcal{S}=(V, E)$, is a multi-edge graph $\left(V^{\prime}, E^{\prime}\right)$, where $V^{\prime}=E$, and the number of edges between two blocks $e, e^{\prime} \in E$ is $\left|e \cap e^{\prime}\right|$. Note that, when $\mathcal{S}$ is linear, the line graph $L(\mathcal{S})$ is a simple graph. The dual set system $\mathcal{S}^{*}$ of $\mathcal{S}$ is a set system with $I\left(\mathcal{S}^{*}\right)=I(\mathcal{S})^{T}$.

Now we give some definitions commonly used in graph theory. The set system $\left(V,\left(\begin{array}{l}V \\ 2\end{array}\right)\right)$ is called a complete graph, denoted by $K_{n}$ if $|V|=n$. A graph is called an $r$-partite graph if its vertices can be partitioned into $r$-parts, such that two vertices are adjacent only when they belong to different parts. It is further called complete r-partite if every two vertices from different parts are adjacent. If a complete $r$-partite graph has parts of size $m_{i}, i \in[r]$, then we denote it by $K_{m_{1}, m_{2}, \ldots, m_{r}}$. A Turán graph $T(n, r)$ is an $n$-vertex complete $r$-partite graph, such that all parts are of size either $\left\lceil\frac{n}{r}\right\rceil$ or $\left\lfloor\frac{n}{r}\right\rfloor$. 
The adjacency matrix $A(G)$ of a graph $G=(V, E)$ is a $|V| \times|V|$ matrix whose rows and columns are indexed by $V$, such that $A(G)_{i, j}=1$ if $\{i, j\} \in E$ and 0 else. The neighborhood of a vertex $x$, denoted by $N(x)$, consists of all vertices $y$ that are adjacent to $x$. A cycle in a graph $G$ is a connected 2-regular subgraph of $G$. Denote $\mathcal{C}_{n}=\left(v_{1}, v_{2}, \ldots, v_{n}\right)$ a cycle with edges $v_{i} \sim v_{i+1}, i \in[n-1]$ and $v_{n} \sim v_{1}$. The girth of a graph is the length of its shortest cycle. An independent set of $G=(V, E)$ is a set of pairwise nonadjacent vertices. A perfect matching of $G$ is a set of disjoint edges that cover all vertices. A graph $G$ is said to be 1-factorable if $E$ can be partitioned into perfect matchings.

\section{FR codes based on set systems}

Given an $(n, \alpha, \rho)$ FR code $\mathcal{C}$, the incidence matrix $I(\mathcal{C})$ is an $n \times \theta$ binary matrix with $n \alpha=\theta \rho$, where rows are indexed by the nodes of the FR code, columns are indexed by the symbols of outer MDS codeword, and the entry $I(\mathcal{C})_{i, j}$ is defined as follows:

$$
I(\mathcal{C})_{i, j}= \begin{cases}1 & \text { if node } i \text { contains symbol } j \\ 0 & \text { otherwise. }\end{cases}
$$

Note that each row of $I(\mathcal{C})$ has exactly $\alpha$ ones and each column has exactly $\rho$ ones. It is easy to see that a $\rho$-uniform $\alpha$-regular set system $\mathcal{S}$ of order $n$ gives an $(n, \alpha, \rho)$ FR code $\mathcal{C}$ such that $I(\mathcal{C})=I(\mathcal{S})$. Since the transpose of $I(\mathcal{S})$ can be viewed as the incidence matrix of the dual of $\mathcal{S}$, which is an $\alpha$-uniform $\rho$-regular set system of order $n \alpha / \rho$, thus it also gives an $(n \alpha / \rho, \rho, \alpha)$ FR code $\mathcal{C}^{\prime}$ such that $I\left(\mathcal{C}^{\prime}\right)=I(\mathcal{S})^{T}$.

\section{A NEW MODEL OF ACCESS-BALANCING FR CODES}

Given a regular uniform set system, it can build an FR code as in Section [I-C, where a node indexed by $x$ stores the content consisting of the indices of blocks containing the point $x$. Here, the indices of the blocks can be viewed as chunks of information, that is, symbols of the outer MDS codeword $\left(y_{1}, y_{2}, \cdots, y_{\theta}\right)$. The labels of chunks $y_{1}, y_{2}, \cdots, y_{\theta}$ are directly proportional to their popularities and hence their access frequencies. It has been shown that the access frequencies of chunks experimentally obey Zipf law [3], where the $i$-th most popular chunk has access frequency $1 / i^{\beta}$ for some $\beta>0$. To simplify our model, we assume that the labels of $y_{1}, y_{2}, \cdots, y_{\theta}$ are consecutive integers in $[\theta]$, and discuss the case for Zipf law in the section of Conclusion. The overall popularity of a node amounts to the sum of the labels of chunks stored on the node. To make sure the access request as even as possible among all nodes, the authors in [10] proposed a chunk placement strategy, by what they referred to MaxMinSum placement. The MaxMinSum problem was stated as a point labeling problem for the dual design, which maximizes the minimum sum of block points for all blocks. They solved this problem for Steiner triple systems and their dual [10]. Here, we restate this problem for FR codes directly, i.e., a block labeling problem.

Problem 3.1: [10] Given a $\rho$-uniform $\alpha$-regular set system $\mathcal{S}=(V, E)$ of order $n$ with $V=\left\{v_{1}, v_{2}, \cdots, v_{n}\right\}$ and $E=$ $\left\{e_{1}, e_{2}, \cdots, e_{\theta}\right\}$, where $\theta=n \alpha / \rho$, the problem of constructing a MaxMinSum $(n, \alpha, \rho)$ FR code from $\mathcal{S}$ is equivalent to finding a labeling of blocks in $E$, i.e., a bijection $\sigma$ from $E$ to $[\theta]$, such that the access-minsum

$$
\operatorname{MinSum}\left(\mathcal{S}_{\sigma}\right):=\left\|I(\mathcal{S})\left(\sigma\left(e_{1}\right), \sigma\left(e_{2}\right), \ldots, \sigma\left(e_{\theta}\right)\right)^{T}\right\|_{\mathbb{L}^{m i n}}
$$

is maximized. Here, $\|\mathbf{x}\|_{\mathbb{L}^{\text {min }}}:=\min \left\{x_{i}\right\}$ for $\mathbf{x}=\left(x_{1}, x_{2}, \ldots, x_{n}\right)^{T}$.

In [10], the authors also provided MinMaxSum model, which minimizes the access-maxsum

$$
\operatorname{Max} \operatorname{Sum}\left(\mathcal{S}_{\sigma}\right):=\left\|I(\mathcal{S})\left(\sigma\left(e_{1}\right), \sigma\left(e_{2}\right), \ldots, \sigma\left(e_{\theta}\right)\right)^{T}\right\|_{\mathbb{L}^{\max }},
$$

where $\|\mathbf{x}\|_{\mathbb{L}_{\text {max }}}:=\max \left\{x_{i}\right\}$ for $\mathbf{x}=\left(x_{1}, x_{2}, \ldots, x_{n}\right)^{T}$. However, these two models can not be optimized simultaneously in general. For example, let $\mathcal{C}$ be the FR code based on $K_{4}$ with nodes $\{1,2,3,4\}$ and six symbols, there are two labelings $\sigma_{1}$ and $\sigma_{2}$ for $\mathcal{C}$ as follows:

$$
\begin{aligned}
\sigma_{1}: & \\
& \sigma_{1}(12)=3, \sigma_{1}(13)=1, \sigma_{1}(14)=6, \\
& \sigma_{1}(23)=5, \sigma_{1}(24)=2, \sigma_{1}(34)=4, \\
& \operatorname{MinSum}\left(\mathcal{S}_{\sigma_{1}}\right)=\left\|(10,10,10,12)^{T}\right\|_{\mathbb{L}^{\text {min }}}=10, \\
& \operatorname{MaxSum}\left(\mathcal{S}_{\sigma_{1}}\right)=\left\|(10,10,10,12)^{T}\right\|_{\mathbb{L}^{\text {max }}}=12 . \\
\sigma_{2}: & \\
& \sigma_{2}(12)=3, \sigma_{2}(13)=1, \sigma_{2}(14)=5, \\
& \sigma_{2}(23)=6, \sigma_{2}(24)=2, \sigma_{2}(34)=4, \\
& \operatorname{MinSum}\left(\mathcal{S}_{\sigma_{2}}\right)=\left\|(9,11,11,11)^{T}\right\|_{\mathbb{L}^{\text {min }}}=9, \\
& \operatorname{MaxSum}\left(\mathcal{S}_{\sigma_{2}}\right)=\left\|(9,11,11,11)^{T}\right\|_{\mathbb{L}^{\text {max }}}=11 .
\end{aligned}
$$

By easy computation, we see that $\sigma_{1}$ achieves the maximum access-minsum, while $\sigma_{2}$ achieves the minimum access-maxsum. This means $\sigma_{2}$ is not an optimal labeling under the MaxMinSum model. Now we look at the variance of popularities among all nodes, i.e., the value $\sum_{i=1}^{\theta}\left(p_{i}-\bar{p}\right)^{2}$, where $p_{i}$ is the overall popularity of the $i$ th node, and $\bar{p}$ is the average popularity of all nodes. It is clear that $I(\mathcal{S})\left(\sigma\left(e_{1}\right), \sigma\left(e_{2}\right), \ldots, \sigma\left(e_{\theta}\right)\right)^{T}=\left(p_{1}, p_{2}, \ldots, p_{\theta}\right)^{T}$. Then both $\sigma_{1}$ and $\sigma_{2}$ in the above example 
provide the same level of access-balancing FR codes when minimizing the variance of popularities. That is, the MaxMinSum model does not capture all good labelings with access-balancing property.

Let's look at another example of the FR code based on $K_{8}$. By computer search, we find two optimal labelings $\sigma_{1}$ and $\sigma_{2}$ under the MaxMinSum model, but $\sigma_{2}$ is clearly better than $\sigma_{1}$ for the access-balancing property when you consider their variances.

$$
\begin{array}{ll}
\sigma_{1}: & \\
& \sigma_{1}(12)=1, \sigma_{1}(13)=2, \sigma_{1}(14)=3, \sigma_{1}(15)=14, \sigma_{1}(16)=26, \sigma_{1}(17)=27, \sigma_{1}(18)=28, \\
& \sigma_{1}(23)=4, \sigma_{1}(24)=10, \sigma_{1}(25)=17, \sigma_{1}(26)=21, \sigma_{1}(27)=23, \sigma_{1}(28)=25, \sigma_{1}(34)=24, \\
& \sigma_{1}(35)=22, \sigma_{1}(36)=15, \sigma_{1}(37)=16, \sigma_{1}(38)=18, \sigma_{1}(45)=20, \sigma_{1}(46)=19, \sigma_{1}(47)=12, \\
& \sigma_{1}(48)=13, \sigma_{1}(56)=8, \sigma_{1}(57)=11, \sigma_{1}(58)=9, \sigma_{1}(67)=7, \sigma_{1}(68)=5, \sigma_{1}(78)=6, \\
& \text { MinSum }\left(\mathcal{S}_{\sigma_{1}}\right)=\left\|(101,101,101,101,101,101,102,104)^{T}\right\|_{\mathbb{L}^{\text {min }}}=101 . \\
\sigma_{2}: & \\
& \sigma_{2}(12)=1, \sigma_{2}(13)=2, \sigma_{2}(14)=3, \sigma_{2}(15)=14, \sigma_{2}(16)=26, \sigma_{2}(17)=27, \sigma_{2}(18)=28, \\
& \sigma_{2}(23)=4, \sigma_{2}(24)=10, \sigma_{2}(25)=17, \sigma_{2}(26)=21, \sigma_{2}(27)=23, \sigma_{2}(28)=25, \sigma_{2}(34)=24, \\
& \sigma_{2}(35)=22, \sigma_{2}(36)=15, \sigma_{2}(37)=16, \sigma_{2}(38)=18, \sigma_{2}(45)=20, \sigma_{2}(46)=19, \sigma_{2}(47)=12, \\
& \sigma_{2}(48)=13, \sigma_{2}(56)=9, \sigma_{2}(57)=11, \sigma_{2}(58)=8, \sigma_{2}(67)=7, \sigma_{2}(68)=5, \sigma_{2}(78)=6, \\
& \operatorname{MinSum}\left(\mathcal{S}_{\sigma_{2}}\right)=\left\|(101,101,101,101,101,102,102,103)^{T}\right\|_{\mathbb{L}^{\text {min }}}=101 .
\end{array}
$$

Since the variance of popularities among all nodes is definitely a key factor that should be considered for the access-balancing property, we introduce a new model to capture this property in next subsection.

\section{A. The MinVar model}

In this subsection, we present a new chunk placement strategy, which we call MinVar placement. The MinVar placement is to minimize the variance of popularities among all nodes of the FR code by relabeling chunks. We formalize the problem as follows.

Problem 3.2: Given a $\rho$-uniform $\alpha$-regular set system $\mathcal{S}=(V, E)$ of order $n$ with $V=\left\{v_{1}, v_{2}, \cdots, v_{n}\right\}$ and $E=$ $\left\{e_{1}, e_{2}, \cdots, e_{\theta}\right\}$, where $\theta=n \alpha / \rho$, the problem of constructing a $\operatorname{Min} \operatorname{Var}(n, \alpha, \rho)$ FR code from $\mathcal{S}$ is equivalent to finding a labeling of blocks in $E$, i.e., a bijection $\sigma$ from $E$ to $[\theta]$, such that the access-variance

$$
\operatorname{Var}\left(\mathcal{S}_{\sigma}\right):=\left\|I(\mathcal{S})\left(\sigma\left(e_{1}\right), \sigma\left(e_{2}\right), \ldots, \sigma\left(e_{\theta}\right)\right)^{T}-(\bar{a}, \bar{a}, \ldots, \bar{a})^{T}\right\|_{\mathbb{L}^{2}}
$$

is minimized. Here, $\bar{a}$ equals the average popularity $\frac{\rho \theta(\theta+1)}{2 n}=\frac{\alpha(\theta+1)}{2}$ and $\|\mathbf{x}\|_{\mathbb{L}^{2}}:=\sum_{i=1}^{n} x_{i}^{2}$ for $\mathbf{x}=\left(x_{1}, x_{2}, \ldots, x_{n}\right)^{T}$.

Let $p_{\sigma}=\left(p_{1}, p_{2}, \ldots, p_{n}\right):=I(\mathcal{S})\left(\sigma\left(e_{1}\right), \sigma\left(e_{2}\right), \ldots, \sigma\left(e_{\theta}\right)\right)^{T}$. By the definition of $I(\mathcal{S})$, the $i$-th component $p_{i}$ is the total popularity of node $i$, which equals to $\sum_{j: v_{i} \in e_{j}} \sigma\left(e_{j}\right)$. So the value of $\operatorname{Var}\left(\mathcal{S}_{\sigma}\right) / n$ can be viewed as the variance of the distribution of the popularities of $n$ nodes, which measures how far the popularity of each node is from the mean. Since the parameter $n$ is fixed, minimizing the value of $\operatorname{Var}\left(\mathcal{S}_{\sigma}\right)$ can yield a kind of evenly access request FR code.

Denote

$$
\operatorname{Min} \operatorname{Var}(\mathcal{S})=\min _{\sigma} \operatorname{Var}\left(\mathcal{S}_{\sigma}\right)
$$

Then a MinVar FR code is an FR code based on $\mathcal{S}$ equipped with a labeling $\sigma$ such that $\operatorname{Var}\left(\mathcal{S}_{\sigma}\right)=\operatorname{MinVar}(\mathcal{S})$. If $\mathcal{S}$ is a regular graph, then we find that $\operatorname{Min} \operatorname{Var}(\mathcal{S})=0$ if and only if the graph is supermagic. We review some known results about magic labeling of graphs in the next subsection.

\section{B. Magic labeling}

The concept of magic labeling in graph theory was introduced by Sedláček [22] in 1963, when considering the notion of magic squares in number theory. After that, Stewart studied various problems to label the edges of a graph in [27] and [28]. Given a connected graph $G=(V, E)$, and an injective mapping $\sigma$ from $E$ into positive integers, let

$$
\sigma^{*}(v):=\sum_{e \in E: v \in e} \sigma(e)
$$

If $\sigma^{*}(v)=\lambda$ for all $v \in V$, then we say $\sigma$ is a magic labeling of $G$ for an index $\lambda$. Further if $\{\sigma(e): e \in E\}$ consists of consecutive positive integers, then we say $\sigma$ is supermagic. A graph $G$ is supermagic (magic) whenever there exists a supermagic (magic) labeling of $G$.

There is by now a considerable number of papers published on magic and supermagic graphs, see for example [12], [13], [15], [16], [23], [25], [29]. Regular supermagic graphs were extended to degree-magic graphs if the set of labels is [|E|], and $\sigma^{*}(v)=\operatorname{deg}(v)(1+|E|) / 2$ for all $v \in V[1],[2]$. Note that if $G$ is a regular graph, then $G$ is supermagic if and only if it is degree-magic [1]. We refer the readers to [14] for comprehensive references. 
If $G$ is a supermagic (or degree-magic) regular graph, then the supermagic labeling $\sigma$ satisfies that $V a r\left(G_{\sigma}\right)=0$ by comparing the definition of $\operatorname{Var}\left(G_{\sigma}\right)$ and degree-magic labeling. In other words, a supermagic regular graph can construct a MinVar FR code with zero access-variance.

Ivančo [15] gave a characterization of all supermagic regular complete multipartite graphs, which we summarize as follows. Note that regular complete multipartite graphs are just regular Turán graphs.

Theorem 3.3: [15] The Turán graph $T(n, r)$ with $r \mid n$ and $r \geq 2$, is supermagic if and only if one of the following conditions is satisfied:

(1) $n=r$, i.e., $K_{n}$, with $n=2$, or $n \geq 6$ and $n \not \equiv 0(\bmod 4)$;

(2) $n=2 r \geq 6$, i.e., $T(2 r, r)$ with $r \geq 3$;

(3) $n \geq 3 r$, except when $r \equiv 0(\bmod 4)$ and $\frac{n}{r}$ is odd.

Theorem 3.4: [26] The Turán graph $T(n, r)$ with $r \mid n$ and $r \geq 2$, gives a $k$-optimal $(n, \alpha, 2)$ FR code for all $k \leq \alpha$, where $\alpha=(r-1) \frac{n}{r}$, hence gives an optimal $(n, \alpha, 2)$ FR code.

Combining Theorems 3.3 and 3.4 we immediately get the following result.

Corollary 3.5: Let $r \geq 2, r \mid n$ and $\alpha=(r-1) \frac{n}{r}$. There exists an optimal MinVar $(n, \alpha, 2)$ FR code with zero access-variance if one of the following conditions is satisfied:

(1) $n=r=2$, or $n=r \geq 6$ and $n \not \equiv 0(\bmod 4)$;

(2) $n=2 r \geq 6$;

(3) $n \geq 3 r$, except when $r \equiv 0(\bmod 4)$ and $\frac{n}{r}$ is odd.

Question 3.6: When $n=r \equiv 0(\bmod 4)$, i.e., complete graphs with order divisible by four, or $r \equiv 0(\bmod 4)$ and $\frac{n}{r}$ is odd, what is $\operatorname{Min} \operatorname{Var}(\mathcal{S})$ for $\mathcal{S}=T(n, r)$ ?

In [26], the authors showed that graphs with large girth can produce optimal FR codes.

Theorem 3.7: [26] If there exists an $\alpha$-regular graph of girth $g$, then there exists a $k$-optimal $(n, \alpha, 2)$ FR code for all $k \leq g-1$, where $n$ is the number of vertices of the graph. Further if $g \geq \alpha+1$, then the FR code is optimal.

Question 3.8: For regular graphs with large girth, what is the minimum access-variance?

Note that Problem 3.2 can be viewed as an extension of the magic labeling problem when a graph is not supermagic, which provides a reference to measure how far a labeling is from a supermagic labeling.

\section{An equivalent problem}

Problem 3.2 is to find a block labeling of a set system to minimize the access-variance. Here, we present an equivalent problem, which looks for a good vertex labeling of the line graph.

For convenience, we always assume that $\mathcal{S}=(V, E)$ is a $\rho$-uniform $\alpha$-regular set system of order $n$ with $V=\left\{v_{1}, v_{2}, \cdots, v_{n}\right\}$ and $E=\left\{e_{1}, e_{2}, \cdots, e_{\theta}\right\}$, where $\theta=n \alpha / \rho$ and $I(\mathcal{S})$ is the incidence matrix whose rows and columns are indexed by $V$ and $E$. Let $\mathbf{1}_{n}$ be the all-1 column vector of length $n$. Then

$$
I(\mathcal{S}) \cdot \mathbf{1}_{\theta}=\alpha \cdot \mathbf{1}_{n}, \text { and } \mathbf{1}_{n}^{T} \cdot I(\mathcal{S})=\rho \cdot \mathbf{1}_{\theta}^{T}
$$

Denote $M(\mathcal{S}):=I(\mathcal{S})^{T} \cdot I(\mathcal{S})$, then

$$
M(\mathcal{S})_{(i, j)}= \begin{cases}\rho & \text { if } i=j \\ \left|e_{i} \cap e_{j}\right| & \text { if } i \neq j\end{cases}
$$

If $\mathcal{S}$ is a linear set system, then $M(\mathcal{S})=\rho I_{\theta}+A(L(\mathcal{S}))$, where $I_{\theta}$ is an identity matrix of order $\theta$, and $A(L(\mathcal{S}))$ is the adjacency matrix of the line graph $L(\mathcal{S})$. Clearly, $L(\mathcal{S})$ is a $d$-regular graph with $\theta$ vertices, where $d=\rho(\alpha-1)$, i.e., $A(L(\mathcal{S}))$ has $d$ ones in each row and each column. If $\mathcal{S}$ is not a linear set system, $A(L(\mathcal{S}))$ is the adjacency matrix of a multi-graph $L(\mathcal{S})$, where vertex $e_{i}$ is adjacent to vertex $e_{j}$ with exactly $\left|e_{i} \cap e_{j}\right|$ parallel edges.

Lemma 3.9: Given a $\rho$-uniform $\alpha$-regular linear set system $\mathcal{S}=(V, E)$ of order $n$ and an edge labeling $\sigma$ with $\sigma\left(e_{j}\right)=i_{j}$, the access-variance

$$
\operatorname{Var}\left(\mathcal{S}_{\sigma}\right)=\left(i_{1}, i_{2}, \cdots, i_{\theta}\right) A(L(\mathcal{S}))\left(i_{1}, i_{2}, \cdots, i_{\theta}\right)^{T}+c
$$

where $c=c(\theta, \rho, \alpha)$ is a constant.

Proof. Because $\sigma\left(e_{j}\right)=i_{j}$ is a bijection, we have

$$
\sum_{j=1}^{\theta} i_{j}=\sum_{j=1}^{\theta} j=\frac{\theta^{2}+\theta}{2}, \text { and } \sum_{j=1}^{\theta} i_{j}{ }^{2}=\sum_{j=1}^{\theta} j^{2}=\frac{\theta(\theta+1)(2 \theta+1)}{6}
$$


By $I(\mathcal{S}) \cdot \mathbf{1}_{\theta}=\alpha \cdot \mathbf{1}_{n}$, and $\mathbf{1}_{\theta}^{T} \cdot A(L(\mathcal{S}))=\rho(\alpha-1) \mathbf{1}_{\theta}$, we have

$$
\begin{aligned}
\operatorname{Var}\left(\mathcal{S}_{\sigma}\right) & =\left\|I(\mathcal{S})\left(i_{1}-\frac{\bar{a}}{\alpha}, i_{2}-\frac{\bar{a}}{\alpha}, \cdots, i_{\theta}-\frac{\bar{a}}{\alpha}\right)^{T}\right\|_{\mathbb{L}^{2}} \\
& =\left(i_{1}-\frac{\bar{a}}{\alpha}, i_{2}-\frac{\bar{a}}{\alpha}, \cdots, i_{\theta}-\frac{\bar{a}}{\alpha}\right) M(\mathcal{S})\left(i_{1}-\frac{\bar{a}}{\alpha}, i_{2}-\frac{\bar{a}}{\alpha}, \cdots, i_{\theta}-\frac{\bar{a}}{\alpha}\right)^{T} \\
& \triangleq\left(i_{1}, i_{2}, \cdots, i_{\theta}\right) M(\mathcal{S})\left(i_{1}, i_{2}, \cdots, i_{\theta}\right)^{T}+c_{1} \\
& \triangleq\left(i_{1}, i_{2}, \cdots, i_{\theta}\right) A(L(\mathcal{S}))\left(i_{1}, i_{2}, \cdots, i_{\theta}\right)^{T}+c_{1}+c_{2} \\
& \triangleq \sum_{e_{k} \cap e_{l} \neq \emptyset} i_{k} i_{l}+c,
\end{aligned}
$$

where

$$
\begin{aligned}
c_{1} & =\left(-\frac{\bar{a}}{\alpha} \mathbf{1}_{\theta}^{T}\right) M(\mathcal{S})\left(-\frac{\bar{a}}{\alpha} \mathbf{1}_{\theta}\right)-2\left(i_{1}, i_{2}, \cdots, i_{\theta}\right) M(\mathcal{S})\left(\frac{\bar{a}}{\alpha} \mathbf{1}_{\theta}\right) \\
& =\frac{\bar{a}^{2}}{\alpha^{2}} \mathbf{1}_{\theta}^{T} \rho \alpha \mathbf{1}_{\theta}-2 \frac{\bar{a}}{\alpha}\left(i_{1}, i_{2}, \cdots, i_{\theta}\right) \rho \alpha \mathbf{1}_{\theta} \quad\left(\text { Since } M(\mathcal{S}) \mathbf{1}_{\theta}=\rho \alpha \mathbf{1}_{\theta}\right) \\
& =\frac{\bar{a}^{2}}{\alpha} \rho \theta-2 \bar{a} \rho \sum_{j=1}^{\theta} j=\bar{a} \rho \theta\left(\frac{\bar{a}}{\alpha}-(\theta+1)\right) \\
& =-\frac{\bar{a} \rho \theta(\theta+1)}{2}, \quad\left(\text { Since } \frac{\bar{a}}{\alpha}=\frac{\theta+1}{2}\right)
\end{aligned}
$$

and $c_{2}=\rho \sum_{i=1}^{\theta} i^{2}$. Then $c=c_{1}+c_{2}=\frac{\rho \theta(\theta+1)(2 \theta+1)}{6}-\frac{\rho \alpha \theta(\theta+1)^{2}}{4}$.

From Lemma 3.9, we only need to consider the line graph of $\mathcal{S}$ in Problem 3.2 So it is natural to propose the following optimization problem about graph labeling of vertices, which is equivalent to Problem 3.2 if we let $G=L(\mathcal{S})$.

Problem 3.10: Given a $d$-regular graph $G$ with $\theta$ vertices, $v_{1}, v_{2}, \cdots, v_{\theta}$, find a weight function $f: V(G) \rightarrow[\theta]$, which is a bijection, such that $\mathcal{M}(f):=\sum_{v_{i} \sim v_{j}} f\left(v_{i}\right) f\left(v_{j}\right)$ is minimized. Denote $\mathcal{M}(G)=\min _{f} \mathcal{M}(f)$, and call it the MinPS of $G$ (which stands for the minimum product sum). Note that each edge in $\mathcal{M}(f)$ is computed only once in the summation.

Now we give the motivation of why we study Problem 3.10 for union of complete or Turán graphs, cycles in Section IV As in Questions 3.6 and 3.8, we are interested in the value of $\operatorname{MinVar}(\mathcal{S})$ when $\mathcal{S}$ is a Turán graph or a graph with large girth. Alternatively, we can estimate the corresponding MinPS of $G=L(\mathcal{S})$ in Problem 3.10 For example, when $\mathcal{S}$ is a cycle (a graph with large girth), $L(\mathcal{S})$ is also a cycle, whose MinPS can be determined as in Section IV-C However, for general Turán graphs $T(n, r)$, it is not easy to determine the MinPS of $L(\mathcal{S})$, even when $\mathcal{S}=T(n, n)$ with $n \equiv 0(\bmod 4)$, i.e., the complete graph $K_{n}$. For this case, we will decompose $K_{n}$ into a Turán graph $T(n, n / 4)$, which is supermagic, and a union of $n / 4$ disjoint $K_{4}$ 's, whose line graph is a union of $n / 4$ disjoint Turán graphs $T(6,3)$. When determining the MinPS for the union of Turán graphs, we need to determine it for the union of complete graphs as in Lemma 4.5. Besides, our results for these graphs can give solutions to Problem 3.2 for some FR codes, see the examples given in the beginning of each subsection of Section IV.

Before closing this section, we give a general upper bound on the MinPS of $G$, which will be improved in Section $\mathrm{V}$ for $G$ being the line graph of a complete graph.

Lemma 3.11: Given a $d$-regular graph $G$ with $\theta$ vertices, $\mathcal{M}(G) \leq \frac{d(3 \theta+2) \theta(\theta+1)}{24}$.

Proof. We compute the average value of $\mathcal{M}(f)$,

$$
\begin{aligned}
\sum_{\sigma \in S_{\theta}} \mathcal{M}\left(f_{\sigma}\right) & =\sum_{\sigma \in S_{\theta}} \sum_{v_{i} \sim v_{j}} f_{\sigma}\left(v_{i}\right) f_{\sigma}\left(v_{j}\right)=\sum_{v_{i} \sim v_{j}} \sum_{\sigma \in S_{\theta}} f_{\sigma}\left(v_{i}\right) f_{\sigma}\left(v_{j}\right) \\
& =\sum_{v_{i} \sim v_{j}}(\theta-2) ! \sum_{1 \leq a \neq b \leq \theta} a b=\sum_{v_{i} \sim v_{j}}(\theta-2) !\left(\left(\sum_{i=1}^{\theta} i\right)^{2}-\sum_{i=1}^{\theta} i^{2}\right) \\
& =\frac{d(3 \theta+2) \theta^{2}\left(\theta^{2}-1\right)}{24}(\theta-2) ! .
\end{aligned}
$$

Hence, $\mathcal{M}(G) \leq \frac{d(3 \theta+2) \theta(\theta+1)}{24}$.

\section{Determination of MinPS in Problem 3.10}

In this section, we focus on solving Problem 3.10 for several classes of graphs, such as disjoint union of complete graphs, Turán graphs and cycles. For convenience, let $[a, b]$ denote the set of integers $\{a, a+1, \ldots, b\}$ for any integers $a \leq b$, and $[1, b]$ is abbreviated to $[b]$.

Before solving Problem 3.10, we illustrate the relation between the MinVar problem and the MinPS problem for linear set systems and their dual in Figure 1. 


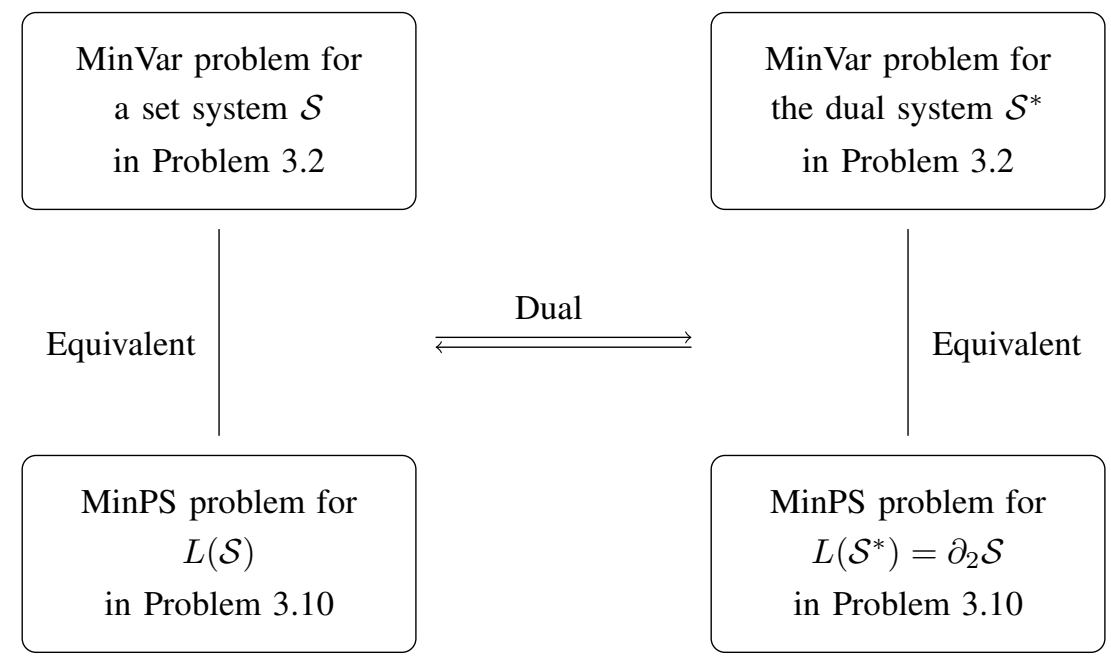

Fig. 1. Relationship of the MinVar and MinPS problems for linear set systems and their dual. Any two blocks in $\mathcal{S}^{*}$ are incident if and only if the corresponding points in $\mathcal{S}$ are in the same block, thereby $L\left(\mathcal{S}^{*}\right)=\partial_{2} \mathcal{S}$.

\section{A. Union of complete graphs}

]Let $m K_{r}$ be a graph that is a disjoint union of $m$ copies of $K_{r}$. In this subsection, we consider the MinPS of $m K_{r}$, whose value will be used in Lemma 4.5 for a union of Turán graphs $m T(n, r)$.

When $m=1$, it is just a complete graph $K_{r}$. It is easy to see that the value $\mathcal{M}(f)$ is a constant for all labelings $f$, and $\mathcal{M}\left(K_{r}\right)=\sum_{1 \leq i<j \leq r} i j$. FR codes $\mathcal{S}$ with $L(\mathcal{S})$ being complete graphs exist, for example when $\mathcal{S}$ is a $2-\left(q^{2}+q+1, q+1,1\right)$ design (i.e., symmetric design [9]), in which every pair of blocks intersect in exactly one point.

Lemma 4.1: For $m \geq 2$, the MinPS value $\mathcal{M}\left(m K_{r}\right)$ can be achieved by the labeling satisfying that the label sums for each copy are as equal as possible.

Proof. Given a vertex labeling $f$ of $m K_{r}$, let $V_{i}$ be the set of labels of the $i$ th copy of $K_{r}$, then $\left|V_{i}\right|=r$ and $\cup_{i=1}^{m} V_{i}=[m r]$. Then

$$
\begin{aligned}
\mathcal{M}(f) & =\sum_{i=1}^{m} \sum_{u<v \in V_{i}} u v=\sum_{i=1}^{m} \frac{1}{2}\left(\left(\sum_{u \in V_{i}} u\right)^{2}-\sum_{u \in V_{i}} u^{2}\right) \\
& =\frac{1}{2} \sum_{i=1}^{m}\left(\sum_{u \in V_{i}} u\right)^{2}-\frac{1}{2} \sum_{i=1}^{m r} i^{2} \\
& \geq \frac{m}{2}\left(\frac{\sum_{i=1}^{m r} i}{m}\right)^{2}-\frac{m r(m r+1)(2 m r+1)}{12} \\
& =\frac{m r^{2}(m r+1)^{2}}{8}-\frac{m r(m r+1)(2 m r+1)}{12} .
\end{aligned}
$$

The inequality holds when $\sum_{u \in V_{i}} u$ is the same for all $i \in[m]$. This can be achieved except when $r$ is odd and $m$ is even (in this case, $\frac{\sum_{i=1}^{m r} i}{m}$ is not an integer), for which the minimum value can be achieved if $\sum_{u \in V_{i}} u$ are almost the same for all $i \in[m]$, i.e., pairwise difference is at most one.

Remark 4.2: The value of $\mathcal{M}\left(m K_{r}\right)$ in Lemma 4.1 can be achieved by the following constructions.

(1) When $r$ is even, for each $i \in[m]$, let

$$
V_{i}=\left[\frac{(i-1) r}{2}+1, \frac{i r}{2}\right] \bigcup\left[m r+1-\frac{i r}{2}, m r-\frac{(i-1) r}{2}\right]:=V_{i}^{(r)} .
$$

Then $\sum_{u \in V_{i}} u=\frac{(r m+1) r}{2}$ for all $i$, and $\mathcal{M}\left(m K_{r}\right)=\frac{m r^{2}(m r+1)^{2}}{8}-\frac{m r(m r+1)(2 m r+1)}{12}$.

(2) When $m$ and $r>1$ are both odd, let

$$
V_{i}=V_{i}^{(r-3)} \bigcup\left\{(r-3) m+i, \frac{(2 r-3) m-1}{2}+i, r m+2-2 i\right\}, i \in\left[\frac{m+1}{2}\right]
$$

and

$$
V_{i}=V_{i}^{(r-3)} \bigcup\left\{(r-3) m+i, \frac{(2 r-5) m-1}{2}+i,(r+1) m+2-2 i\right\}, i \in\left[\frac{m+3}{2}, m\right] .
$$


Then $\sum_{u \in V_{i}} u=\frac{(r m+1) r}{2}$ for all $i$, and $\mathcal{M}\left(m K_{r}\right)=\frac{m r^{2}(m r+1)^{2}}{8}-\frac{m r(m r+1)(2 m r+1)}{12}$.

(3) When $r>1$ is odd and $m$ is even, let

$$
V_{i}=V_{i}^{(r-3)} \bigcup\left\{(r-3) m+i, \frac{(2 r-3) m}{2}+i, r m+2-2 i\right\}, i \in\left[\frac{m}{2}\right]
$$

and

$$
V_{i}=V_{i}^{(r-3)} \bigcup\left\{(r-3) m+i, \frac{(2 r-5) m}{2}+i,(r+1) m+1-2 i\right\}, i \in\left[\frac{m}{2}+1, m\right]
$$

Then

$$
\sum_{u \in V_{i}} u= \begin{cases}\frac{(r m+1) r+1}{2}, & i \in\left[\frac{m}{2}\right] \\ \frac{(r m+1) r-1}{2}, & i \in\left[\frac{m}{2}+1, m\right]\end{cases}
$$

Hence, $\mathcal{M}\left(m K_{r}\right)=\frac{m r^{2}(m r+1)^{2}+m}{8}-\frac{m r(m r+1)(2 m r+1)}{12}$.

\section{B. Union of Turán graphs}

Next, we determine the MinPS of a Turán graph $T(n, r)$ with $r \mid n$. FR codes based on the dual transversal designs $\operatorname{TD}(r, n / r)$ [9] have the line graph $T(n, r)$ for any $r \mid n$. We first provide a simple but very useful remark below, which will be frequently used in our proofs.

Remark 4.3: Suppose that $f$ is a weight function of $G$ achieving the MinPS. For every vertex $v$, denote $f(N(v)):=$ $\sum_{u \in N(v)} f(u)$. If $v_{i}$ and $v_{j}$ are not adjacent and $f\left(v_{i}\right)<f\left(v_{j}\right)$, then we claim that $f\left(N\left(v_{i}\right)\right) \geq f\left(N\left(v_{j}\right)\right)$. In fact, if $f\left(N\left(v_{i}\right)\right)<f\left(N\left(v_{j}\right)\right)$, then we switch the weight value between $v_{i}$ and $v_{j}$, and obtain a new weight function $f^{\prime}$, for which $f^{\prime}\left(N\left(v_{i}\right)\right)=f\left(N\left(v_{i}\right)\right), f^{\prime}\left(N\left(v_{j}\right)\right)=f\left(N\left(v_{j}\right)\right)$ and $f^{\prime}\left(v_{i}\right)=f\left(v_{j}\right), f^{\prime}\left(v_{j}\right)=f\left(v_{i}\right)$. But

$$
\begin{aligned}
\mathcal{M}\left(f^{\prime}\right)-\mathcal{M}(f) & =f^{\prime}\left(v_{i}\right) f^{\prime}\left(N\left(v_{i}\right)\right)+f^{\prime}\left(v_{j}\right) f^{\prime}\left(N\left(v_{j}\right)\right)-f\left(v_{i}\right) f\left(N\left(v_{i}\right)\right)-f\left(v_{j}\right) f\left(N\left(v_{j}\right)\right) \\
& =f\left(v_{i}\right)\left(f\left(N\left(v_{j}\right)\right)-f\left(N\left(v_{i}\right)\right)\right)+f\left(v_{j}\right)\left(f\left(N\left(v_{i}\right)\right)-f\left(N\left(v_{j}\right)\right)\right) \\
& =\left(f\left(N\left(v_{i}\right)\right)-f\left(N\left(v_{j}\right)\right)\right)\left(f\left(v_{j}\right)-f\left(v_{i}\right)\right)<0,
\end{aligned}
$$

which is a contradiction to the fact that $\mathcal{M}(f)=\mathcal{M}(G)$.

Lemma 4.4: Let $r \mid n$ and $r \geq 2$. Then the MinPS of $T(n, r)$

$$
\mathcal{M}(T(n, r))=\sum_{1 \leq j<j^{\prime} \leq r}\left(\sum_{k=(j-1) n / r+1}^{j n / r} k\right)\left(\sum_{l=\left(j^{\prime}-1\right) n / r+1}^{j^{\prime} n / r} l\right) .
$$

Proof. Let $m=n / r \geq 2$ (when $m=1$, the graph is a complete graph, which is trivial). Suppose that $G=(V, E)$ is a Turán graph $T(n, r)$, with $V=V_{1} \cup V_{2} \cup \cdots \cup V_{r}$ and $\left|V_{i}\right|=m$. Given a weight function $f$, denote $f\left(V_{i}\right)=\sum_{v \in V_{i}} f(v)$ and $f(V)=$ $\sum_{v \in V} f(v)$, then $\mathcal{M}(f)=\sum_{1 \leq i<j \leq r} f\left(V_{i}\right) f\left(V_{j}\right)$. Without loss of generality, we assume that $f\left(V_{1}\right) \leq f\left(V_{2}\right) \leq \cdots \leq f\left(V_{r}\right)$.

We claim that the weight function which minimizes $\mathcal{M}(f)$ has the property that $f\left(V_{i}\right)=\sum_{j=(i-1) m+1}^{i m} j, i \in[r]$, that is, the labels of vertices in $V_{i}$ are $(i-1) m+1,(i-1) m+2, \ldots, i m$.

We prove it by contradiction. Assume that for some $i<i^{\prime}$, there exists a label $x \in V_{i}$ and a label $y \in V_{i^{\prime}}$ satisfying $x>y$. By switching the labels $x$ and $y$, we get a new weight function $f^{\prime}$, for which

$$
\begin{aligned}
\mathcal{M}\left(f^{\prime}\right)-\mathcal{M}(f) & =\sum_{1 \leq i<j \leq r} f^{\prime}\left(V_{i}\right) f^{\prime}\left(V_{j}\right)-\sum_{1 \leq i<j \leq r} f\left(V_{i}\right) f\left(V_{j}\right) \\
& =\left(f\left(V_{i}\right)-x+y\right)\left(f\left(V_{i^{\prime}}\right)-y+x\right)-f\left(V_{i}\right) f\left(V_{i^{\prime}}\right) \\
& =\left(f\left(V_{i}\right)-f\left(V_{i^{\prime}}\right)\right)(x-y)-(x-y)^{2} .
\end{aligned}
$$

Since $f\left(V_{i^{\prime}}\right) \geq f\left(V_{i}\right)$ and $x>y$, we have $\mathcal{M}\left(f^{\prime}\right)<\mathcal{M}(f)$, a contradiction.

Now we consider the graph $m T(n, r)$ with $r \mid n$, which is a disjoint union of copies of $T(n, r)$. The MinPS of $m T(n, r)$ will be used in the estimation of $\operatorname{MinVar}\left(K_{4 r}\right)$ in Lemma 5.2

Lemma 4.5: The MinPS of $m T(n, r)$ with $r \mid n$ can be determined by the MinPS of $m K_{r}$.

Proof. Let $l=n / r \geq 2$ and $V_{i j}$ be the set of labels of vertices from the $i$ th part of the $j$ th copy of $T(n, r), i \in[r]$ and $j \in[m]$. For any labeling $f$, we have $\left|V_{i j}\right|=l$ and $\bigcup_{i \in[r], j \in[m]} V_{i j}=[m n]$. Suppose that $f$ minimizes $\mathcal{M}(f)$. Let $V_{j}=\cup_{i \in[r]} V_{i j}$. By the proof of Lemma 4.4 we know that for each $j, V_{1 j}$ is the set of the smallest $l$ integers from $V_{j}, V_{2 j}$ is the set of the smallest $l$ integers from $V_{j} \backslash V_{1 j}$, and so on. For convenience, we denote this property by $\mathcal{P}$.

We claim that for any two different sets $V_{i j}$ and $V_{i^{\prime} j^{\prime}}$, all integers in $V_{i j}$ are either smaller than each integer in $V_{i^{\prime} j^{\prime}}$, or greater than each integer in $V_{i^{\prime} j^{\prime}}$. That is to say, each $V_{i j}$ must be exactly a set $U_{t}=[l(t-1)+1, l t]$ for some $t \in[m r]$. We 
prove the claim by contradiction. Suppose that there exist two sets $V_{i j}$ and $V_{i^{\prime} j^{\prime}}$, and integers $x, y \in V_{i j}$ and $z, w \in V_{i^{\prime} j^{\prime}}$, such that $x>z$ and $y<w$. By property $\mathcal{P}$, we have $j \neq j^{\prime}$. Let $F_{j}=\sum_{s \neq i} V_{s j}$ and $F_{j^{\prime}}=\sum_{a \neq i^{\prime}} V_{a j^{\prime}}$. Suppose that $F_{j} \geq F_{j^{\prime}}$. Let $f^{\prime}$ be a new labeling by switching $x$ and $z$. Then

$$
\begin{aligned}
\mathcal{M}\left(f^{\prime}\right)-\mathcal{M}(f) & =\left(z F_{j}+x F_{j^{\prime}}\right)-\left(x F_{j}+z F_{j^{\prime}}\right) \\
& =(z-x)\left(F_{j}-F_{j^{\prime}}\right) \leq 0 .
\end{aligned}
$$

Thus, by switching $x$ and $z$, the value of $\mathcal{M}(f)$ does not increase. Continuing this operation, we can have all integers in $V_{i j}$ are smaller than those in $V_{i^{\prime} j^{\prime}}$. Repeating this step for any such pair $V_{i j}$ and $V_{i^{\prime} j^{\prime}}$, eventually each $V_{i j}$ becomes some $U_{t}$.

By the above claim, we can assume that each $V_{i j}$ is a set $U_{t}$ for some $t$. Now we compute $\mathcal{M}(f)$. Assume that $V_{i j}=U_{t}$ and $V_{i^{\prime} j}=U_{t^{\prime}}$ in the following equation.

$$
\begin{aligned}
\mathcal{M}(f) & =\sum_{j \in[m]} \sum_{1 \leq i<i^{\prime} \leq r}\left(\sum_{u \in V_{i j}} u\right)\left(\sum_{u \in V_{i^{\prime} j}} u\right) \\
& =\sum_{j \in[m]} \sum_{1 \leq i<i^{\prime} \leq r} \frac{l(2 l t-l+1)}{2} \times \frac{l\left(2 l t^{\prime}-l+1\right)}{2} \\
& =\left(\begin{array}{l}
r \\
2
\end{array}\right) \frac{m l^{2}(1-l)^{2}}{4}+l^{3}(1-l) \sum_{j \in[m]} \sum_{1 \leq i<i^{\prime} \leq r} \frac{t+t^{\prime}}{2}+l^{4} \sum_{j \in[m]} \sum_{1 \leq i<i^{\prime} \leq r} t t^{\prime} \\
& =\left(\begin{array}{l}
r \\
2
\end{array}\right) \frac{m l^{2}(1-l)^{2}}{4}+\frac{l^{3}-l^{4}}{4} r m(r m+1)(r-1)+l^{4} \mathcal{M}(\bar{f}),
\end{aligned}
$$

where $\bar{f}$ is the induced vertex labeling for $m K_{r}$. By Remark 4.2, we can find the optimal labeling $\bar{f}$ with $\mathcal{M}(\bar{f})=\mathcal{M}\left(m K_{r}\right)$, from which we can deduce the optimal labeling $f$ for $m T(n, r)$.

\section{Cycles}

Large cycles are graphs with large girth, whose line graphs are also themselves. Now we consider the MinPS of the cycle $C_{\theta}$. Denote $\mathcal{M}_{\theta}=\mathcal{M}\left(\mathcal{C}_{\theta}\right)$.

Lemma 4.6: For any $\theta \geq 3$, we have $\mathcal{M}_{\theta+2} \geq \mathcal{M}_{\theta}+\theta^{2}+4 \theta+5$.

Proof. We prove it by contradiction. Suppose that there exists a labeling $f$ of $\mathcal{C}_{\theta+2}$ such that $\mathcal{M}(f)$ is minimized and $\mathcal{M}(f)<\mathcal{M}_{\theta}+\theta^{2}+4 \theta+5$. From $f$, if we can deduce a labeling $f^{\prime}$ of $\mathcal{C}_{\theta}$ satisfying $\mathcal{M}\left(f^{\prime}\right)<\mathcal{M}_{\theta}$, then we are done. We split the proof into three cases.

Case 1: Suppose that in $\mathcal{C}_{\theta+2}$, we have a segment with labels $\cdots x \theta+21 y \cdots$. We operate this cycle in the following two steps.

(S1) Delete the two vertices with labels 1 and $\theta+2$, and connect the two vertices with labels $x$ and $y$. The value of $\mathcal{M}(f)$ becomes $M=\mathcal{M}(f)-x(\theta+2)-(\theta+2)-y+x y$.

(S2) Change each label $l$ by $l-1$ of vertices in the cycle of length $\theta$. Then we obtain a labeling $f^{\prime}$ of a cycle $\mathcal{C}_{\theta}$.

Now we compute $\mathcal{M}\left(f^{\prime}\right)$. Note that in (S1), $M=\sum_{u \sim v} u v$, and each $u \in[2, \theta+1]$ appears twice in this summation. Since $u v=(u-1)(v-1)+u+v-1$, we have

$$
M=\sum_{u \sim v}(u-1)(v-1)+2 \sum_{i=2}^{\theta+1} i-\theta=\mathcal{M}\left(f^{\prime}\right)+\theta^{2}+2 \theta .
$$

So after (S2), we have

$$
\begin{aligned}
\mathcal{M}\left(f^{\prime}\right) & =\mathcal{M}(f)-x(\theta+2)-(\theta+2)-y+x y-\theta^{2}-2 \theta \\
& <\mathcal{M}_{\theta}+\theta^{2}+4 \theta+5-x(\theta+2)-(\theta+2)-y+x y-\theta^{2}-2 \theta \\
& =\mathcal{M}_{\theta}+(x-1)(y-\theta-2)+1 .
\end{aligned}
$$

Since $y<\theta+2$ and $x \geq 2$, we have $\mathcal{M}\left(f^{\prime}\right)<\mathcal{M}_{\theta}$, which is a contradiction.

Case 2: Suppose that in $\mathcal{C}_{\theta+2}$, we have a segment with labels $\cdots x \theta+2 z 1 y \cdots$. Delete the two vertices with labels 1 and $\theta+2$, and connect $x z$ and $y z$. The value of $\mathcal{M}(f)$ becomes

$$
M=\mathcal{M}(f)-(x+z)(\theta+2)-(y+z)+x z+y z .
$$

Applying (S2) in Case 1, we obtain a labeling $f^{\prime}$ of a cycle $\mathcal{C}_{\theta}$. Similar to Case 1, we have

$$
\begin{aligned}
\mathcal{M}\left(f^{\prime}\right) & =M-\theta^{2}-2 \theta \\
& =\mathcal{M}(f)-(x+z)(\theta+2)-(y+z)+x z+y z-\theta^{2}-2 \theta \\
& <\mathcal{M}_{\theta}+\theta^{2}+4 \theta+5-(x+z)(\theta+2)-(y+z)+x z+y z-\theta^{2}-2 \theta \\
& =\mathcal{M}_{\theta}+(x-2)(z-\theta-2)+(z-1)(y-1)-z \theta .
\end{aligned}
$$


Since $y, z<\theta+2$ and $x \geq 2$, we have $\mathcal{M}\left(f^{\prime}\right)<\mathcal{M}_{\theta}$, which is a contradiction.

Case 3: Suppose that in $\mathcal{C}_{\theta+2}$, we have a segment with labels $\cdots x \theta+2 z \cdots w 1 y \cdots$. We claim that $z$ and $w$ are not adjacent. Otherwise, $f(N(z))=\theta+2+w$ and $f(N(1))=y+w$, thus $f(N(1))<f(N(z))$, which contradicts to Remark 4.3. Similarly, $x$ and $y$ can not be adjacent. Suppose $w>y$ (the case when $w<y$ is similar), and a label $a$ is next to $w$ on the left, i.e., the segment is $\cdots a w 1 y \cdots$ around 1 . We operate this cycle in the following two steps.

(T1) Delete the two vertices with labels 1 and $w$, and connect the two vertices with labels $a$ and $y$. The value of $\mathcal{M}(f)$ becomes $M=\mathcal{M}(f)-a w-w-y+a y$.

(T2) Change each label $l>w$ by $l-2$, and each label $l<w$ by $l-1$ in the cycle of length $\theta$. Then we obtain a labeling $f^{\prime}$ of a cycle $\mathcal{C}_{\theta}$.

Since $(u-2)(v-2)=u v-2(u+v)+4,(u-1)(v-2)=u v-2 u-v+2$ and $(u-1)(v-1)=u v-(u+v)+1$, we have

$$
\begin{aligned}
\mathcal{M}\left(f^{\prime}\right) & =M-\sum_{u \sim v: u, v>w}(2 u+2 v-4)-\sum_{u \sim v: u<w<v}(2 u+v-2)-\sum_{u \sim v: u, v<w}(u+v-1) \\
& =M-4 \sum_{i=w+1}^{\theta+2} i-2 \sum_{i=2}^{w-1} i+\sum_{u \sim v: u<w<v}(v-u+2)+\sum_{u \sim v: u, v>w} 4+\sum_{u \sim v: u, v<w} 1 \\
& \triangleq M-2(\theta+w+3)(\theta-w+2)-(w+1)(w-2)+S,
\end{aligned}
$$

where $S$ is the sum of the last three terms. Now we compute $\mathcal{M}\left(f^{\prime}\right)-\mathcal{M}_{\theta}$, which is less than

$$
T(w):=\theta^{2}+4 \theta+5-a w-w-y+a y-2(\theta+w+3)(\theta-w+2)-(w+1)(w-2)+S .
$$

We claim that $T(w)<0$ for any $w$, thus $\mathcal{M}\left(f^{\prime}\right)<\mathcal{M}_{\theta}$, a contradiction. We prove it by upper bounding the value of $S$. Note that when $u \sim v$, and $u<w<v$, one has $v-u+2 \geq 4$. Hence, the more crossing edges there are between $[2, w-1]$ and $[w+1, \theta+2]$, the bigger $S$ it is. Based on the value of $w$, we split into two cases.

If $w \leq \frac{\theta}{2}+2$, then $|[2, w-1]| \leq|[w+1, \theta+2]|$. Therefore,

$$
\begin{aligned}
S & \leq 2 \sum_{i=\theta-w+5}^{\theta+2} i-2 \sum_{i=2}^{w-1} i+4(w-2)+4(\theta-2 w+4) \\
& =(2 \theta-w+11)(w-2)-(w+1)(w-2)+4(\theta-2 w+4) \\
& =-2 w^{2}+(2 \theta+6) w-4,
\end{aligned}
$$

which is maximized when $w=\frac{\theta}{2}+2$. Hence,

$$
\begin{aligned}
T(w) & \leq-w^{2}+(2 \theta+8) w-(\theta+3)^{2}+a(y-w)-y \\
& =a(y-w)-y-\frac{\theta^{2}}{4}+3<0 .
\end{aligned}
$$

If $w \geq \frac{\theta}{2}+2$, then $|[2, w-1]| \geq|[w+1, \theta+2]|$. Therefore,

$$
\begin{aligned}
S & \leq 2 \sum_{i=w+1}^{\theta+2} i-2 \sum_{i=2}^{\theta-w+3} i+4(\theta-w+2)+(2 w-\theta-4) \\
& =(\theta+w+7)(\theta-w+2)-(\theta-w+5)(\theta-w+2)+(2 w-\theta-4) \\
& =-2 w^{2}+(2 \theta+4) w+\theta,
\end{aligned}
$$

which is maximized when $w=\theta+1$. Then

$$
\begin{aligned}
T(w) & \leq-w^{2}+(2 \theta+5) w-\theta^{2}-5 \theta-5+(a-1)(y-w) \\
& =(a-1)(y-w)-1<0 .
\end{aligned}
$$

Next, we show that the lower bound of the MinPS of $C_{\theta}$ in Lemma 4.6 can be achieved.

Lemma 4.7: For any $\theta \geq 3$, we have $\mathcal{M}_{\theta+2}=\mathcal{M}_{\theta}+\theta^{2}+4 \theta+5$. Hence, $\mathcal{M}_{2 k+1}=\left(4 k^{3}+12 k^{2}+14 k+3\right) / 3$ and $\mathcal{M}_{2 k+2}=\left(4 k^{3}+18 k^{2}+29 k+12\right) / 3$ for each $k \geq 1$.

Proof. We prove a stronger statement that for each $\theta \geq 3$, there exists a labeling $f$ for $\mathcal{C}_{\theta}$ achieving $\mathcal{M}_{\theta}$ such that the labels 1 and $\theta$ are adjacent.

For $\theta=3, \mathcal{M}(f)$ is constant and $\mathcal{M}_{3}=11$, and two labels 1 and 3 are adjacent. For $\theta=4$, by Lemma 4.4, $\mathcal{M}_{4}=21$, and two labels 1 and 4 are adjacent. Assume that for any cycles of length no more than $\theta$, our statement is true, then we prove that it is true for $\theta+2$. By assumption, there exists a labeling $f^{\prime}$ with $\mathcal{M}\left(f^{\prime}\right)=\mathcal{M}_{\theta}$ for $\mathcal{C}_{\theta}$ such that the labels 1 and $\theta$ are adjacent. Now increase each label of $f^{\prime}$ by one, then we have two labels 2 and $\theta+1$ adjacent. By inserting two vertices with 
labels $1, \theta+2$ between 2 and $\theta+1$, we have a segment as $\cdots 2 \theta+21 \theta+1 \cdots$, and obtain a labeling $f$ of a cycle $\mathcal{C}_{\theta+2}$. It is easy to check that

$$
\begin{aligned}
\mathcal{M}(f) & =\mathcal{M}_{\theta}+2 \sum_{i=1}^{\theta} i+\theta-2(\theta+1)+2(\theta+2)+(\theta+2)+(\theta+1) \\
& =\mathcal{M}_{\theta}+\theta^{2}+4 \theta+5 .
\end{aligned}
$$

Hence, $\mathcal{M}_{\theta+2} \leq \mathcal{M}_{\theta}+\theta^{2}+4 \theta+5$. By Lemma 4.6, we have proved our statement. The exact values can be easily computed from $\mathcal{M}_{3}, \mathcal{M}_{4}$, and the recursion.

We further show that the labeling in Lemma 4.7 for $\mathcal{C}_{\theta}$ also maximizes the access-minsum in Problem 3.1

Theorem 4.8: There exists a labeling for $\mathcal{C}_{\theta}$ which is optimal for both Problem 3.1 and Problem 3.2

Proof. Let $\mathcal{S}_{\theta}=(V, E)$ be the set system whose line graph is $\mathcal{C}_{\theta}$. Note that $\mathcal{S}_{\theta}$ is also a cycle of length $\theta$. The vertex-labeling $f$ of $\mathcal{C}_{\theta}$ in Lemma 4.7 naturally induces an edge labeling $\sigma$ of $\mathcal{S}_{\theta}$, which minimizes the access-variance of $\mathcal{S}_{\theta}$ in the MinVar model.

Now we claim that it also maximizes the access-minsum for the MaxMinSum model by induction on $\theta$. It is easy to get that the maximum access-minsum of $\mathcal{S}_{\theta}$ is at most $\theta$. When $\theta=3,4$, it is trivially true. Assume that for all $\theta$ or less the claim is true, let us consider $\theta+2$. By assumption, the edge labeling $\sigma^{\prime}$ induced by $f^{\prime}$ in Lemma 4.7 for cycle $C_{\theta}$ maximizes the access-minsum for $\mathcal{S}_{\theta}$, i.e., for each edge $v_{i} \sim v_{j}$ in $C_{\theta}$, we have $f^{\prime}\left(v_{i}\right)+f^{\prime}\left(v_{j}\right) \geq \theta$. By the proof of Lemma 4.7 the labeling $f$ looks like $\cdots 2 \theta+21 \theta+1 \cdots$, which is obtained from $f^{\prime}$ by increasing each label by one, then deleting the edge $2 \sim \theta+1$ and adding three new edges $2 \sim \theta+2, \theta+2 \sim 1,1 \sim \theta+1$. It is clear that for all edges $v_{i} \sim v_{j}$ in $C_{\theta+2}$, we have $f\left(v_{i}\right)+f\left(v_{j}\right) \geq \theta+2$. Hence, the induced edge labeling $\sigma$ maximizes the access-minsum of $\mathcal{S}_{\theta+2}$.

\section{Estimate $\operatorname{MinVaR}(\mathcal{S})$ in $\operatorname{Problem} 3.2$}

In this section, we estimate the value of $\operatorname{Min} \operatorname{Var}(\mathcal{S})$ in $\operatorname{Problem} 3.2$ for some set systems $\mathcal{S}$.

Lemma 5.1: Let $H_{1}=\left(V, E_{1}\right)$ and $H_{2}=\left(V, E_{2}\right)$ be two regular graphs on the same vertex set. If $E_{1} \cap E_{2}=\emptyset$ and $H_{1}$ is supermagic, then the minimum access-variance of $G=\left(V, E_{1} \cup E_{2}\right)$ satisfies

$$
\operatorname{Min} \operatorname{Var}(G) \leq \operatorname{MinVar}\left(H_{2}\right) .
$$

Proof. We prove it by giving an edge labeling $\sigma$ of $G$. For graph $H_{1}$, we label the edges by set $\left[\left|E_{2}\right|+1,\left|E_{1}\right|+\left|E_{2}\right|\right]$ such that $\sigma^{*}(v)$ is constant for all $v \in V$. This can be done since $H_{1}$ is supermagic. Then label edges in $H_{2}$ by $\left[1,\left|E_{2}\right|\right]$ such that the variance is $\operatorname{Min} \operatorname{Var}\left(H_{2}\right)$. It is easy to compute that $\operatorname{Var}\left(G_{\sigma}\right)=\operatorname{Min} \operatorname{Var}\left(H_{2}\right)$.

Now consider the access-variance of $K_{4 r}$, which is an open case in Question 3.6 Further, FR codes based on complete graphs yield the first class of MBR codes which have the additional property of exact repair by transfer [20], [24]. To estimate $\operatorname{Min} \operatorname{Var}\left(K_{4 r}\right)$, we view $K_{4 r}$ as a disjoint union of $r K_{4}$ and $T(4 r, r)$. By Theorem 3.3, $T(4 r, r)$ is supermagic. By Lemma 5.1, we have $\operatorname{Min} \operatorname{Var}\left(K_{4 r}\right) \leq \operatorname{MinVar}\left(r K_{4}\right)$. Note that the line graph of $r K_{4}$ is $r T(6,3)$, for which the MinPS has been determined in Lemma 4.5. By the connection of values $\mathcal{M}(r T(6,3))$ and $\operatorname{MinVar}\left(r K_{4}\right)$ in Lemma 3.9, we can give an upper bound of $\operatorname{Min} \operatorname{Var}\left(K_{4 r}\right)$.

Lemma 5.2: For any positive integer $r$, we have

$$
\operatorname{Min} \operatorname{Var}\left(K_{4 r}\right) \leq \begin{cases}3 r, & r \text { is odd } \\ 7 r, & r \text { is even }\end{cases}
$$

Proof. By setting parameters in Lemma 3.9 as $\rho=2, \alpha=3, \theta=6 r$, and parameters in Lemma 4.5 as $m=r, n=6, r=3$, the value of $\operatorname{Min} \operatorname{Var}\left(K_{4 r}\right)$ is upper bounded by $32 M-72 r^{2}-18 r+c$, where $c=-r(6 r+1)(30 r+7)$ is determined by Lemma 3.9, and

$$
M= \begin{cases}\frac{45 r^{3}+36 r^{2}+7 r}{8}, & r \text { is odd; } \\ \frac{45 r^{3}+36 r^{2}+8 r}{8}, & r \text { is even }\end{cases}
$$

is determined by Lemma 4.1 for $r K_{3}$.

Combining Lemma 3.9 and the averaging upper bound in Lemma 3.11, we have $\operatorname{MinVar}\left(K_{4 r}\right)=O\left(r^{7}\right)$, which is greatly improved to $O(r)$ by Lemma 5.2. It is easy to derive a lower bound to show that $\operatorname{Min} \operatorname{Var}\left(K_{4 r}\right)=\Theta(r)$. In fact, the average popularity of all nodes in $K_{4 r}$ is $(4 r-1)\left(8 r^{2}-2 r+1\right) / 2$, whose fractional part is 0.5 . Since the total popularity for each node is an integer, we have $\operatorname{Min} \operatorname{Var}\left(K_{4 r}\right) \geq 4 r \times(0.5)^{2}=r$. We raise the following question.

Question 5.3: Whether the upper bound in Lemma 5.2 is tight? It is true for $r=1$. 
Now we consider Turán graphs. Let $G=L(T(n, r))$ be the line graph of a Turán graph with $r \mid n$ and $r \geq 2$. By Question 3.6 we only need to deal with the case $r \equiv 0(\bmod 4)$ and $\frac{n}{r}$ is odd. Chetwynd and Hilton gave the following property of regular graphs.

Theorem 5.4: [7] Let $G$ be a $d$-regular graph of $2 n$ vertices and $d \geq \frac{12}{7} n$. Then $G$ is 1-factorable.

By Theorem 5.4, $T(n, r)$ is 1-factorable when $r \geq 7, n$ is even and $r \mid n$. Note that a perfect matching in $T(n, r)$ will be an independent set of $L(T(n, r))$. Let $m=\frac{(r-1) n}{r}$, and $d=2(m-1)$. Then $G=L(T(n, r))=(V, E)$ is an $m$-partite $d$-regular graph, where $V=V_{1} \cup V_{2} \cup \cdots \cup V_{m}$ with $\left|V_{i}\right|=\frac{n}{2}, i \in[m]$. Further, for each $i \neq j \in[m]$, the subgraph induced by $V_{i} \cup V_{j}$ is a 2-regular graph.

Question 5.5: For every $r \geq 7$ and even $n$ satisfying $r \mid n$, can we determine of MinPS of $G=L(T(n, r))$ ?

\section{CONCLUSION}

Motivated by the DRESS codes and access-balancing problem in distributed storage systems, we propose a new combinatorial model, called MinVar model, which is a problem of labeling blocks of set systems such that the access-variance is minimized (Problem 3.2). This problem can be viewed as a generalization of the magic labeling problem when graphs are not supermagic. We further establish an equivalent problem if the set system is linear, which is a vertex-labeling problem of graphs (Problem 3.10). By solving both problems, we are able to find serval families of optimal FR codes based on special graphs, which have minimum access-variance. Besides their applications in access-balancing issue in distributed storage, we think Problems 3.2 and 3.10 are interesting by themselves and worth further study in the future. Especially, we restate Question 3.6 for more attention.

Question 3.6: When $n=r \equiv 0(\bmod 4)$, or $r \equiv 0(\bmod 4)$ and $\frac{n}{r}$ is odd, what is $\operatorname{Min} \operatorname{Var}(\mathcal{S})$ for $\mathcal{S}=T(n, r)$ ?

We have given an upper bound in Lemma 5.2 for the case $n=r \equiv 0(\bmod 4)$, which we think is tight. For the other case, we only have a weak bound in Lemma 3.11

Next, we briefly discuss the model when the access frequencies of information chunks obey the Zipf law. Using the same notations, Problem 3.10 can be rewritten as follows.

Problem 6.1: Given a $d$-regular graph $G$ with $\theta$ vertices, $v_{1}, v_{2}, \cdots, v_{\theta}$ and some $\beta>0$, find a bijection $\ell: V(G) \rightarrow$ $\left\{1,1 / 2^{\beta}, \ldots, 1 / \theta^{\beta}\right\}$, such that $\overline{\mathcal{M}}(\ell)=\sum_{v_{i} \sim v_{j}} \ell\left(v_{i}\right) \ell\left(v_{j}\right)$ is minimized. Denote the MinPS of $G$ as $\overline{\mathcal{M}}(G)=\min _{\ell} \overline{\mathcal{M}}(\ell)$.

By the similar arguments, we can obtain some parallel results with respect to Problem 6.1. First, when $G=K_{\theta}, \overline{\mathcal{M}}(\ell)$ is a constant for any vertex labeling. For Turán graphs, $\overline{\mathcal{M}}(T(n, r))$ can be achieved when the $k$-th part of vertices is labeled by $\left\{\frac{1}{((k-1) r+1)^{\beta}}, \frac{1}{((k-1) r+2)^{\beta}}, \ldots, \frac{1}{(k r)^{\beta}}\right\}$. However, for union of complete graphs or Turán graphs, we need to solve the following set-partition problem to estimate $\overline{\mathcal{M}}\left(m K_{r}\right)$ and $\overline{\mathcal{M}}(m T(n, r))$.

Question 6.2: Find an equipartition of $\left\{1,1 / 2^{\beta}, \ldots, 1 /(m r)^{\beta}\right\}$ into $m$ subsets $S_{1}, S_{2}, \ldots, S_{m}$ in polynomial time, such that $\sum_{i=1}^{m}\left(\sum_{j \in S_{i}} j\right)^{2}$ is minimized.

Finally, we mention that the upper bounds of the maximum file size $A(n, k, \alpha, \rho)$ of $[(\theta, M), k,(n, \alpha, \rho)]$ DRESS in Eqs. (1) and (2) are still tight in some cases even if we impose the zero access-variance property. Indeed, Corollary 3.5 provides some examples of zero access-variance optimal FR codes. For general FR codes with a limited access-variance, it is interesting that one can improve the upper bounds in Eqs. (11) and (2). However, it is not easy to apply their original proofs in [21] to the access-variance case. We leave this problem for future study.

\section{REFERENCES}

[1] L. Bezegová and J. Ivančo. An extension of regular supermagic graphs. Discrete Mathematics, 310(24):3571-3578, 2010.

[2] L. Bezegová and J. Ivančo. A characterization of complete tripartite degree-magic graphs. Discussiones Mathematicae Graph Theory, 32(2):243-253, 2012.

[3] L. Breslau, P. Cao, L. Fan, G. Phillips, and S. Shenker. Web caching and Zipf-like distributions: Evidence and implications. in Proceedings of the IEEE International Conference on Computer Communications, pages 126-134, 1999.

[4] W. M. Brummond. Kirkman systems that attain the upper bound on the minimum block sum, for access balancing in distributed storage. arXiv preprint arXiv:1906.02157 2019.

[5] Y. M. Chee, C. J. Colbourn, H. Dau, R. Gabrys, A. C. Ling, D. Lusi, and O. Milenkovic. Access balancing in storage systems by labeling partial steiner systems. Designs, Codes and Cryptography, 88(11):2361-2376, 2020.

[6] L. Cherkasova and M. Gupta. Analysis of enterprise media server workloads: access patterns, locality, content evolution, and rates of change. IEEE/ACM Transactions on Networking (TON), 12(5):781-794, 2004

[7] A. G. Chetwynd and A. J. W. Hilton. Regular graphs of high degree are 1-factorizable. Proc. London Math. Soc. (3), 50(2):193-206, 1985.

[8] A. Cidon, S. Rumble, R. Stutsman, S. Katti, J. Ousterhout, and M. Rosenblum. Copysets: Reducing the frequency of data loss in cloud storage. In Presented as part of the $2013\{$ USENIX\} Annual Technical Conference (\{USENIX\}\{ATC\} 13), pages 37-48, 2013.

[9] C. J. Colbourn. CRC handbook of combinatorial designs. CRC press, 2010.

[10] H. Dau and O. Milenkovic. MaxMinSum Steiner systems for access balancing in distributed storage. SIAM Journal on Discrete Mathematics, 32(3):1644$1671,2018$.

[11] A. G. Dimakis, P. B. Godfrey, Y. Wu, M. J. Wainwright, and K. Ramchandran. Network coding for distributed storage systems. IEEE Transactions on Information Theory, 56(9):4539-4551, 2010.

[12] M. Doob. Generalizations of magic graphs. Journal of Combinatorial Theory, Series B, 17(3):205-217, 1974.

[13] M. Doob. Characterizations of regular magic graphs. Journal of Combinatorial Theory, Series B, 25(1):94-104, 1978.

[14] J. Gallian. A dynamic survey of graph labeling (2018). The Electronic Journal of Combinatorics., 2018. 
[15] J. Ivančo. On supermagic regular graphs. Mathematica Bohemica, 125(1):99-114, 2000.

[16] R. Jeurissen. Magic graphs, a characterization. European Journal of Combinatorics, 9(4):363-368, 1988.

[17] O. Olmez and A. Ramamoorthy. Repairable replication-based storage systems using resolvable designs. In 2012 50th Annual Allerton Conference on Communication, Control, and Computing (Allerton), pages 1174-1181. IEEE, 2012.

[18] O. Olmez and A. Ramamoorthy. Fractional repetition codes with flexible repair from combinatorial designs. IEEE Transactions on Information Theory, 62(4):1565-1591, 2016.

[19] S. Pawar, N. Noorshams, S. El Rouayheb, and K. Ramchandran. Dress codes for the storage cloud: Simple randomized constructions. In 2011 IEEE International Symposium on Information Theory Proceedings, pages 2338-2342. IEEE, 2011.

[20] K. Rashmi, N. B. Shah, P. V. Kumar, and K. Ramchandran. Explicit construction of optimal exact regenerating codes for distributed storage. In 2009 47th Annual Allerton Conference on Communication, Control, and Computing (Allerton), pages 1243-1249. IEEE, 2009.

[21] S. El Rouayheb and K. Ramchandran. Fractional repetition codes for repair in distributed storage systems. In 2010 48th Annual Allerton Conference on Communication, Control, and Computing (Allerton), pages 1510-1517. IEEE, 2010.

[22] J. Sedláček. Problem 27. theory of graphs and its applications. In Proc. Symp. Smolenice. Praha, pages 163-164, 1963.

[23] J. Sedláček. On magic graphs. Mathematica Slovaca, 26(4):329-335, 1976.

[24] N. B. Shah, K. V. Rashmi, P. V. Kumar, and K. Ramchandran. Distributed storage codes with repair-by-transfer and nonachievability of interior points on the storage-bandwidth tradeoff. IEEE Transactions on Information Theory, 58(3):1837-1852, 2011.

[25] W. C. Shiu, P. C. B. Lam, and S.-M. Lee. On a construction of supermagic graphs. Journal of Combinatorial Mathematics and Combinatorial Computing, 42:147-160, 2002.

[26] N. Silberstein and T. Etzion. Optimal fractional repetition codes based on graphs and designs. IEEE Transactions on Information Theory, 61(8):41644180, 2015.

[27] B. Stewart. Magic graphs. Canadian Journal of Mathematics, 18:1031-1059, 1966.

[28] B. Stewart. Supermagic complete graphs. Canadian Journal of Mathematics, 19:427-438, 1967.

[29] G. C. Sun, J. Guan, and S.-M. Lee. A labeling algorithm for magic graph. Congressus Numerantium, pages 129-138, 1994.

[30] B. Zhu, K. W. Shum, H. Li, and H. Hou. General fractional repetition codes for distributed storage systems. IEEE Communications Letters, 18(4):660-663, 2014. 